\title{
Properzio 2.31.5-6: stile, costituzione del testo e antichità
}

\author{
Paola PAOLUCCI \\ Università degli Studi di Perugia \\ paolapao71@yahoo.it
}

Recibido: 21 de enero de 2011

Aceptado: 23 de febrero de 2011

\section{RIASSUNTO}

Si sostiene la difesa del testo tràdito dalla tradizione manoscritta potiore nei vv. 5-6 dell'elegia $2.31 \mathrm{di}$ Properzio sulla base di argomenti grammaticali e stilistici, ricostruendo (nell'ambito della storia ecdotica del testo a partire dal XVII sec.) le motivazioni che hanno indotto i filologi ad intervenire sul testo. La lezione tràdita trova, infine, riscontro nelle testimonianze antiquarie circa l'assetto del complesso monumentale del tempio di Apollo sul Palatino.

Palabras clave: Properzio. Constitutio textus. Stile. Antichità. Tempio di Apollo sul Palatino.

Paolucci, P., «Properzio 2.31.5-6: stile, costituzione del testo e antichità», Cuad. Fil. Clás. Estud. Lat. $31.1(2011) 7-25$.

\section{Propertius 2.31.5-6: style, constitutio textus and antiquities}

\begin{abstract}
This paper defends the text transmitted by the most important manuscript tradition of Propertius 2.31.56 , on the grounds of grammatical and stylistic arguments, reconstructing (within the editorial history of the text starting from the XVIIth century) the reasons that led the philologists to emend the text. The transmitted reading can be finally confirmed by the antiquarian witnesses reguarding the arrangement of the monumental complex of Apollo's temple on the Palatine.
\end{abstract}

Keywords: Propertius. Constitutio textus. Style. Antiquities. Apollon Temple on the Palatinus.

PaOlucci, P., «Propertius 2.31.5-6: style, constitutio textus and antiquities», Cuad. Fil. Clás. Estud. Lat. 31.1 (2011) 7-25. 
«Basterebbero questi esempi... per farci dolere che filologi di buona levatura preferiscano fare l'ennesima edizione critica di testi classici che non offrono reali probabilità di avanzamenti sostanziali e trascurino campi molto più fecondi»».

Con queste parole, che invitavano caldamente a coltivare il fertile campo dell'edizione di testi tardoantichi, Scevola Mariotti si apprestava a concludere il Suo intervento Problemi di ecdotica tardoantica al Convegno La cultura in Italia fra tardo antico e alto medioevo, aperto dal quell'indiscusso manager culturale che fu Francesco Della Corte, e tenutosi a Roma nel novembre $1979^{1}$, cioè nello stesso anno in cui uscì l'edizione properziana di Hanslik e ben prima che fosse pubblicata l'edizione properziana di Fedeli (Stuttgart 1984), le due edizioni properziane di Goold (Cambridge, Mass., 1990 e 1999), l'edizione properziana di Luck (Zürich 1996), l'edizione properziana di Giardina (Roma 2005), l'edizione properziana di Simone Viarre (Paris 2005), l'edizione properziana di Heyworth (Oxonii 2007)... tacendo le edizioni properziane parziali e i commenti properziani ${ }^{2}$.

Il ricordo di questa vox clamantis in deserto mi è sovvenuto, allorquando ho avuto occasione di orecchiare l'elegante battuta di Louis Holtz, pronunciata nel Convegno Poesia latina, nuova e-filologia (Perugia, 13-15 settembre 2007) ${ }^{3}$ e nusquam publici iuris facta, il quale asserì di preferir leggere Properzio sul Neapolitanus, affidando poi all'inchiostro, nella stesura definitiva delle Conclusioni degli Atti del medesimo Convegno, la cristallina constatazione seguente: «Il caso di Properzio mi sembra un caso emblematico. La critica si è scatenata e il poeta rischia di scomparire sotto la profusione di correzioni talvolta arbitrarie» (p.411).

In omaggio alla statura e all'alto profilo scientifico ed istituzionale dei filologi testè menzionati, con lo stesso incosciente candore del bambino della celebre fiaba di Andersen (Keiserens Nye Kloeder), vorrei provare a mostrare, librandomi dai miei abituali studi tardoantichi in una fugace incursione a volo sul classico, come un luogo properziano tormentatissimo (e non solo a partire dalle edizioni scientifiche positive, cui spesso -mi è parso- per gli auctores primae classis ci si attiene in modo pressoché esclusivo, dimenticando l'apporto ecdotico ed esegetico della filologia dei secoli XVII e XVIII [a meno che qualcosina, di citazione in citazione, non abbia valicato l'800]) possa essere invece tranquillamente accettato nella lezione tràdita dai codici poziori, previo qualche rudimento di grammatica e di stilistica.

Fornisco testo integrale 4 ed apparato (limitatamente ai versi in esame) dell'elegia 2, 31 dalla laudata edizione di Paolo Fedeli ${ }^{5}$, che fra le recenti è giudicata a buon di-

${ }^{1}$ Atti del Convegno di Roma, C.N.R., 12-16 novembre 1979, Roma 1981, vol. I, pp.323-332, rist. in Mariotti 2000, pp.499-508 (loc. cit. p.506).

${ }^{2}$ E tacendo anche la messe infinita di studi properziani per i quali $c f$. almeno NeTHERCUT 1983, p.1813 sgg. Sugli orientamenti delle moderne edizioni di Properzio $c f$. Viarre 2005, pp.491-499.

${ }^{3}$ Cf. Zurli-Mastandrea 2009.

${ }^{4}$ Senza ignorare i numerosi problemi di unitarietà rispetto all'elegia successiva.

${ }^{5} C f$. Fedeli 1984. 
ritto una delle più solide ed attendibili' ${ }^{6}$, richiamando l'attenzione del lettore sui vv. 5-6 crucibus insigniti $^{7}$, in quanto il v. 5 proporrebbe «un nuovo rompicapo» ${ }^{8}$ :

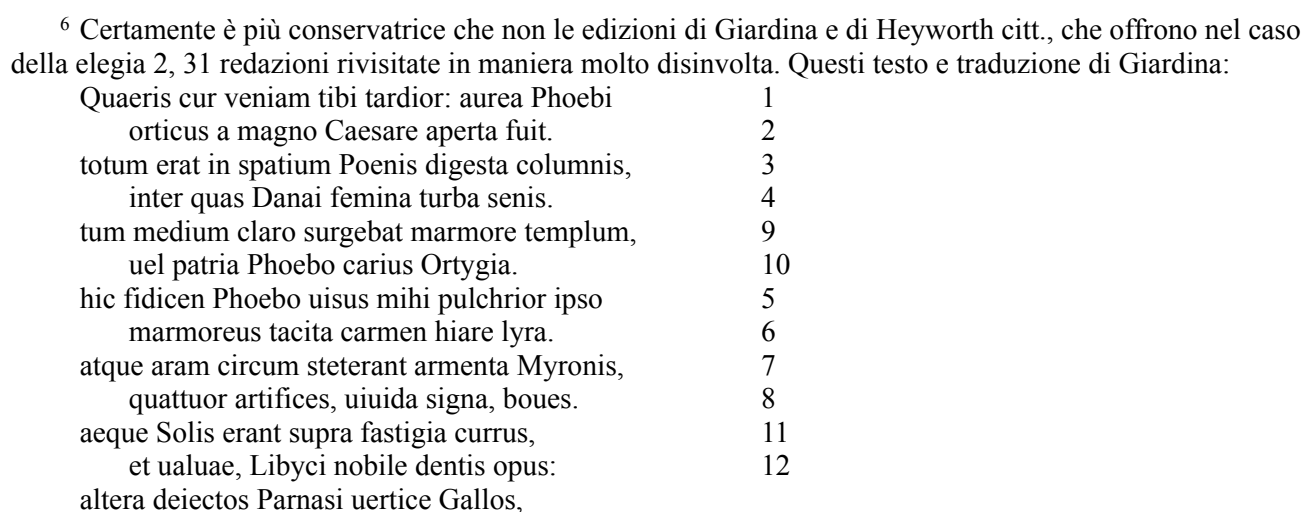
altera deiectos Parnasi uertice Gallos, altera maerebat funera Tantalidos.

deinde inter matrem deus ipse interque sororem $\quad 15$ Pythius in longa carmina ueste sonat.

In adpar. ad v. 5 fidicen Huschke.

«1 Mi chiedi perché vengo da te in ritardo: è stato aperto dal grande Cesare

2 l'aureo portico di Febo.

3 Esso era scandito, in tutta la sua lunghezza, da colonne di marmo africano,

4 in mezzo alle quali stavano effigiate le molte figlie di Danao.

9 poi nel mezzo sorgeva il tempio di Febo, in marmo bianco,

10 un tempio più caro al dio della natia Ortigia.

5 Qui, in un rilievo marmoreo, un suonatore di lira più bello dello stesso Febo

6 mi sembrò aprire la bocca nel canto con il tacito accompagnamento della lira.

7 E intorno all'altare vi era la scultura dovuta a Mirone di quattro buoi,

8 un'opera d'arte, che sembravano vivi.

11 Ugualmente, vi erano il carro del Sole in alto sul frontone,

12 e in basso i due battenti della porta, ottima fattura di avorio libico; uno offriva una mesta descrizione dei Galli respinti giù dalla cima del monte Parnaso, l'altro delle morti dei figli di Niobe, la Tantalide.

15 Poi, lo stesso dio pitico, fra la madre e la sorella,

nel suo lungo mantello suona le sue musiche» (p.215).

E questo il testo di Heyworth:

Quaeris cur veniam tibi tardior? aurea Phoebi porticus a magno Caesare aperta fuit.

tota erat in spatium Poenis digesta columnis, inter quas Danai femina turba senis.

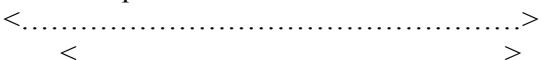

hic equidem Phoebo uisus mihi pulchrior ipso, marmoreus tacita carmen hiare lyra: eqs.

Heyworth 1994, pp.56-59 congettura per il v. 5 illo e teorizza la perdita di un distico prima del medesimo verso; ribadisce le sue convinzioni anche in Heyworth 2009, p.247.

${ }^{7}$ Nell'uso delle cruces in questo luogo il Fedeli si uniformò (prudentemente) al Barber, che tuttavia aveva almeno mostrato la sagacia di divinare dalla lezione eadem di $\mathbf{D V}_{\mathrm{I}}$ la congettura ante aedem Phoebus, da lui medesimo relegata in apparato (e giustamente: ché sembra piuttosto una glossa ad hic); $c f$. Barber $1953 \mathrm{e}$ $1960^{2}$. Le cruces riguardano equidem Phoebo in Butler-Barber 1969.

${ }^{8} C f$. Fedeli 2005, p.876. 
Quaeris, cur veniam tibi tardior? Aurea Phoebi porticus a magno Caesare aperta fuit.

Tanta erat in speciem, Poenis digesta columnis, inter quas Danai femina turba senis.

$\dagger$ Hic equidem Phoebo $†$ visus mihi pulchrior ipso marmoreus tacita carmen hiare lyra;

atque aram circum steterant armenta Myronis, quattuor artifices, vivida signa, boves.

Tum medium claro surgebat marmore templum, et patria Phoebo carius Ortygia,

in quo Solis erat supra fastigia currus et valvae, Libyci nobile dentis opus:

altera deiectos Parnasi vertice Gallos, altera maerebat funera Tantalidos.

Deinde inter matrem deus ipse interque sororem Pythius in longa carmina veste sonat.

5-6 post u. 16 transt. Dousa fil.; cf. tamen Enk II 2, 3965 hic equidem (eadem DV, corr. $\mathrm{V}^{2}$ ) poebo $\omega$ (def. Rothstein I 411) : hic equidem phoebus $\varsigma$ (fort. recte: cf. Enk II 2, 398) : hic Phoebus Phoebo Hoeufft equidem] quidam Markland: fidicen Huschke 6 hiare] habere $\mathrm{DV}^{2}$

«L'imperatore è nudo!»: infatti, dopo seri studi ginnasiali, utili a formare certa dimestichezza con i costrutti sintetici propri delle lingue classiche (tra i primi occorre di sperimentare, ad es., la c.d. comparatio compendiaria $)^{9}$, un bravo giovine, ignaro ancora dei segni diacritici della critica testuale, casserebbe mentalmente le cruces ed interpreterebbe: «qui ${ }^{10}$ in vero quello marmoreo più bello di Febo stesso a me parve cantare a bocca aperta un carme con la lira silenziosa ${ }^{11}$; e poi passerebbe oltre.

E come il giovine del nostro exemplum fictum, in buona sostanza, si sono comportati tutti quegli editori properziani che hanno pubblicato l'elegia nel rispetto della tradizione manoscritta, giudicando che il testo non facesse difficoltà ed evitando di sollevare loro stessi problemi inesistenti: mi riferisco a nomi celeberrimi della filolo-

\footnotetext{
${ }^{9}$ Si concede che nei giovani di tutti i tempi questi costrutti, lontani dalla 'logica' delle lingue moderne, abbiano potuto procurare qualche imbarazzo, tant'è che le grammatiche scolastiche ottocentesche si preoccupavano di 'razionalizzarli', come si legge nella osservazione n. 8 sulla comparatio compendiaria in CLYDE 1856, p.126: «The Greeks frequently used a compendious form of comparison, which is not logically correct,

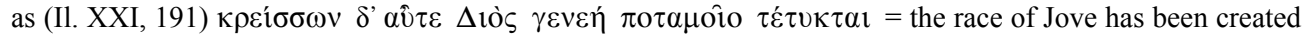

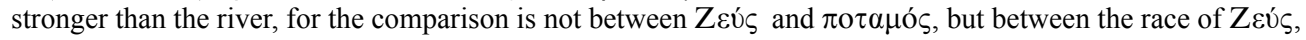
and the race of $\pi$ o $\tau \alpha \mu$ ó $\varsigma$. This illogicality may be avoided by the use of the demonstrative article, which represents the noun understood, as our own that does in 'than that of the river'».

${ }^{10} \mathrm{Hic}$ va senz'altro considerato come avv. di stato in luogo nel senso di in hac porticu, dal momento che i vari distici, come del resto è tipico in ogni poesia ecfrastica, sono scanditi da determinazioni di luogo incipitarie: v. 4 inter quas; v. 5 hic; v. 7 aram circum; v. 9 medium; v. 11 in quo; v. 15 deinde. C'è stato chi ha proposto di considerare hic come pronome riferito a Cesare o a Danao.

${ }^{11} C f$. anche una delle più recenti traduzioni in Katz 2004, p.211: «Here, a marble Phoebus pouring forth a song on silent lyre seemed to me even more beautiful than the god himself»; $c f$. inoltre NAMIA 1973, p.355.
} 
gia dei secoli XVI-XIX, come lo Scaligero ${ }^{12}$, il Broukhusius ${ }^{13}$, il Vulpius ${ }^{14}$, il Burman ${ }^{15}$, il Lachmann (ma nella sua seconda edizione properziana) ${ }^{16}$, il Kuinoel ${ }^{17}$, Fridericus Iacob ${ }^{18}$, Lucianus Mueller ${ }^{19}$, il Nisard ${ }^{20}$, il Rothstein ${ }^{21}$, ma anche ad alcuni rappresentanti della filologia italiana del primo Novecento, come il Ramorino ${ }^{22}$, il Bonazzi $^{23}$, il Martini ${ }^{24}$, oppure ad insigni filologi del XX secolo come Schuster e Dornseiff ${ }^{25}, \mathrm{Camps}^{26}$, Hanslik ${ }^{27}$ ed editori recenti, come Simone Viarre ${ }^{28}$.

L'ellissi $^{29}$ del soggetto ${ }^{30}$ (qui Phoebus) in una comparatio, dove peraltro i due termini di paragone concernono entrambi il medesimo dio (la statua marmorea di Febo ed il dio Febo stesso) - perciò l'uno è agevolmente arguibile dall'altro - e dove l'attributo contribuisce a suggerire il sostantivo, è tratto non atipico nella sintetica lingua latina come anche nella greca ${ }^{31}$ (specie in contesti dialogici e mimetici quale quello presupposto nell'elegia [vd. v. 1 quaeris ${ }^{32}$ ) e soprattutto nella lingua della poesia ${ }^{33}$,

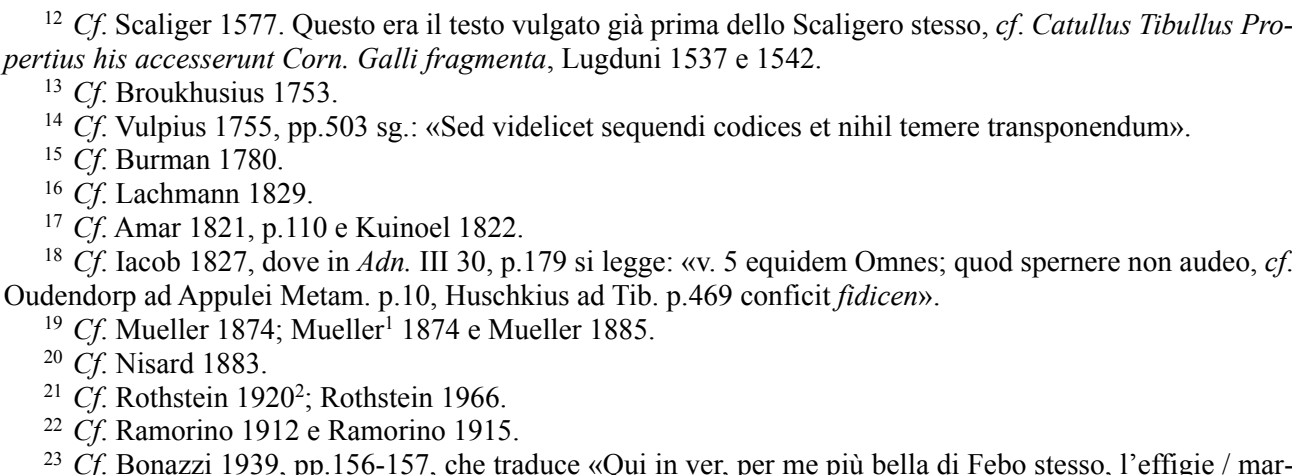
morea spira su muta cetra il canto». Nella successiva edizione (Bonazzi 1951), i vv. 5-6 rimangono secondo la lezione da codice, ma gli altri versi presentano interventi quanto meno discutibili, come causa pro aperta al v. 2; forzata (sul piano grammaticale) e non necessaria è la concordanza di hic del v. 5 con Caesar del v. 2; $c f$. p.171 «Qui Cesare, per me di Febo stesso più bello / marmoreo, spira su muta cetra il canto».

24 Cf. Martini 1931.

${ }^{25}$ Cf. Schuster-Dornseiff 1958.

${ }^{26}$ Cf. p.204 del suo commento al II libro (= Camps 1967).

${ }^{27}$ Cf. Hanslik 1979.

${ }^{28} C f$. Viarre $^{1} 2005$, p. 196 n. 381.

${ }^{29}$ Come è noto, la comparatio compendiaria rientra fra i modi della figura dell'ellissi ed è prioritaria nello stile di Tacito, $c f$. Gudeman 1914, p.CXVIII.

${ }^{30}$ A proposito di «Die Ellipse des Subjectes», specie in presenza di attributo, $c f$. Kühner 1878, p.1039.

${ }^{31}$ Giustamente Kortekaas difende contro gli emendamenti degli altri editori in Historia Apollonii regis Tyri c. 39 nautae Apollonii... melius ceteris navibus, nel senso di melius nautis ceterarum navium, e suppone che il costrutto ellittico possa essere stato presente già nel modello greco ( $c f$. Kortekaas 2007, p.644). Per la lingua greca a confronto con la latina $c f$. anche Middleton 1892, p.59.

32 Parla giustamente di «avvio colloquiale ex abrupto» Gazich 1995, p.156.

${ }^{33}$ L'archetipo degli usi poetici della comparatio compendiaria è costituito da HoM. Il. 17.51 кó $\mu \alpha \iota$

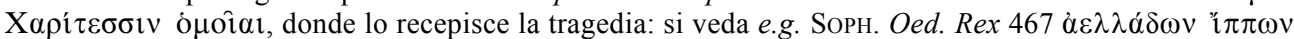
$\sigma \theta \varepsilon v \alpha \rho \omega ́ \tau \varepsilon \rho o v \varphi v \gamma \hat{\alpha} \pi$ ó $\alpha \nu \omega \mu \hat{\alpha} \nu$ considerato esempio di genitivo di comparazione nella comparatio compendiaria e tradotto «to play in flight a foot more strong than (those of) storm-swift horses» (cf. Moorhouse 1982), e Aesch.Pers. $150 \mathrm{sg}$. Ne esibisce esempi anche Orazio, in part. in Carm.2.14.28 Pontificum potiore cenis, che La Penna traduce «migliore di (quello usato nelle) cene dei pontefici» e commenta «Si ha un'ardita comparatio compendiaria»; Carm.2.6.14 sg. ... ubi non Hymetto / mella decedunt..., tradotto dal medesimo 
che elegge la brachilogia a categoria semantica ${ }^{34}$, di cui peraltro Properzio utilizza le molteplici possibilità ${ }^{35}$. Al punto che è nozione elementare di stilistica, vulgata nelle scuole europee sin dall'Ottocento, considerare la «Brachilogy of Comparison» fra gli elementi caratterizzanti la Syntaxis ornata e giudicarla contigua alla Ellipsis intesa come «omission of a word easily understood» ${ }^{36}$. Del resto, notevole è nel luogo in esame anche l'uso della costruzione nominale di visus à̃ò кoıvov̂ (altra figura della brevitas dicendi $)^{37}$ con esse pulchrior (ellittico del verbo sum) e con quel capolavoro di pregnanza espressiva che è carmen hiare, dall'invidiabile fortuna letteraria ${ }^{38}$.

La Penna «il miele non cede (non è inferiore) a quello dell'Imetto» ed annotato «La comparatio è compendiaria» (cf. La Penna 1969, pp.322 e 287 sg.); Carm.3.6.46 aetas parentum, peior avis e Sat.1.1.99-100 at hunc liberta securi / divisit medium, fortissima Tyndaridum. E ne partecipa la lingua poetica di Valerio Flacco, che in Arg.2.226 notaque sonat vox coniuge maior impiega «eine comparatio compendiaria für coniugis voce maior» (cf. KLEYWEGT 1986, p.2482) e in Arg. 1.668 et magno penitus par regia caelo userebbe un'espressione costituente esempio di comparatio compendiaria ove regia par magno caelo equivarrebbe a regia par regiae magni caeli ( $c f$. GALLI 2007, p.346). Ovidio impiega la concentrazione espressiva in tema di canti apollinei, rispetto ai quali Pan avrebbe vantato la propria superiorità, in Met.11.155 ausus Apollineos prae se contemnere cantus («osò disprezzare i canti di Apollo a paragone dei suoi», cf. Scivoletto 2000, p.529), a proposito del qual luogo già Davies 1907, pp.36 sg. osservava: «prae se = prae suis cantibus an istance of comparatio compendiaria the abbreviated comparison, a construction especially adapted to the genius of a synthetic language like Latin or Greek. Their syntax lacks a convenient use of the pronoun like our English 'that of', and they have to adopt either the cumbrous method of repeating the substantive or the compendious form of comparison here noted» e segnalava «for similar brevity» i seguenti altri costrutti: OvID.Met.11.163 post hunc $=$ post huius cantum; 410 fratremque secutis $=$ prodigia fratris sec.; 417 buxoque simillimus. $C f$. inoltre Haupt-Ehwald 1915, ad Met.1.20. In MARTIAL.3.66.1 Par scelus admisit Phariis Antonius armis Fusi 2006, p.424 riconosce la comparatio compendiaria e ne segnala altri casi, che hanno dato adito ad interventi di emendamento inutili.

${ }^{34} C f$. Kroll 1988, pp.47 sgg. (trad. it. di Kroll 1924). Primo esempio di brachilogia è la comparatio compendiaria in Hofmann-Szantyr 2002, p.236.

${ }^{35}$ Cf. Prop.2.1.41-42 dove Caesaris nomen condere in Phrigios avos starebbe per condere carmina de nomine... usque ad (cf. Fedeli 2005, p.80); 2.2.6 ... et incedit vel Iove digna soror, luogo che è spiegato da Shackleton Bailey come equivalente di digna quae sit soror Iovis (cf. Fedeli 2005, p.113: «che lo stile di Properzio possa ammettere una simile brachilogia non sembra affatto da escludere»); 2.14 .25 dove tua ... columna «è espressione brachilogica per tuae aedis columna» (cf. Fedeli 2005, p.431); 2.29a.20 Ad mandatam... domum costituente «un esempio di brachilogia» (cf. Fedeli 2005, p.829) per ad quam iussi sumus deducere o ad domum quae nobis commissa est; 2.24c.40 Ferre formosam per ferre iniurias formosae (cf. Fedeli 2005, p.698). Il celeberrimo Prop.1.9.11 plus in amore valet Mimnermi versus Homero costituirebbe una comparatio compendiaria secondo Riesenweber 2007, p.40: «statt Homeri versu». Crederei che anche in ragione dell'impiego della brachilogia sia stato pronunciato questo verissimo giudizio: «Et est sane difficilis Propertius, cuius sensus ac rationes nisi diligentissimo pariter ac longissimo studio perspicere non possis, sed ut eos quo magis penetraris hoc vehementius te alliciant; plane sicut Tacitus, cum quo in quantum materiae sinit diversitas mirum in modum illi convenit» (cf. Mueller ${ }^{1} 1874$, p.III).

${ }^{36}$ Lo testimonia una lucida ed interessante grammatica conservata nella biblioteca Bodleiana: $c f$. Farrar 1867, p. 85 e $195 \mathrm{sg}$. Essa annovera l'ellittico costrutto nell'ambito dell' 'Order of words and figures of speech» (p.XXI).

${ }^{37}$ Cf. Aken 1884 .

${ }^{38}$ Cf. PRop.3.3.4 tantum operis, nervis hiscere posse meis; 3.17.34 Capripedes calamo Panes hiante canens. Probabilmente - come registravano già i commenti settecenteschi, prima di collegare il nesso con il motivo dell'os magna sonaturum - l'eco del distico elegiaco e della specifica espressione influenza Apul. Flor.1.15 Magna etiam vis aeris vario effigiatu, veterrimo et spectabili opere; vel inde ante aram Bathylli statua a Polycrate tyranno dicata, qua nihil videor effectius cognovisse; quidam Pythagorae eam falso existimant. Adulescens est visenda pulchritudine, crinibus a fronte parili separatu per malas remulsis, pone autem 
Forse, essendo nella manualistica sulla comparatio compendiaria più diffusi gli esempi di elementi sottintesi nel secondo termine di paragone che non nel primo ${ }^{39}$, se Properzio avesse scritto in prosa Phoebus marmoreus visus mihi pulchrior Phoebo ipso (il che non è molto distante da ciò che gli fa dire Hoeufft ${ }^{40}$ ) oppure si fosse attenuto ad un ordo verborum 'impoetico' (marmoreus visus pulchrior Phoebo ipso) forse non si sarebbero usati cruces, emendamenti o trasposizioni di versi ${ }^{41}$; ma, di grazia, il poeta è libero artefice della sua lingua ${ }^{42}$. Ed inoltre la poesia enfatizza per statuto la figura della synchysis ${ }^{43}$ o mixtura verborum, che a me parrebbe magistralmente rappresentata dai vv. 5-6 dell'elegia così come essi sono tramandati dai codici poziori, dove l'agg. marmoreus, ritardato dall'iperbato e suggerente il sogg. sottinteso, persegue quel «gioco straniante» e quella obscuritas ${ }^{44}$ tipici della specifica figura ed adeguati al «modo di parlare eccitato corrispondente alla situazione ${ }^{45}$ dell'elegia in esame. L'iperbole ${ }^{46}$ per la quale la statua marmorea di Apollo sarebbe più bella di Apollo stesso è un altro chiaro indizio dell'enfasi permeante l'intero distico ${ }^{47}$.

Perciò ai fini della costituzione del testo occorre tener presenti le fondamentali parole di Hofmann-Szantyr circa l'ellissi nella sez. 'Semplicità e brevità' della cele-

coma prolixior interlucentem cervicem scapularum finibus obumbrat; cervix suci plena, malae uberes, genae teretes, at medio mento lacullatur; eique prorsus citharoedicus status: deam conspiciens, canenti similis, tunicam picturis variegatam deorsus ad pedes deiectus ipsos, Graecanico cingulo, chlamyde velat utrumque brachium ad usque articulos palmarum, cetera decoris striis dependent; cithara balteo caelato apta strictim sustinetur; manus eius tenerae, procerulae: laeva distantibus digitis nervos molitur, dextra psallentis gestu pulsabulum citharae admovet, ceu parata percutere, cum vox in cantico interquievit; quod interim canticum videtur ore tereti semihiantibus in conatu labellis eliquare. Verum haec quidem statua esto cuiuspiam puberum, qui Polycrati tyranno dilectus Anacreonteum amicitiae gratia cantilat. Cf. inoltre DANTE, Purg. 10.55-60 e soprattutto ARIOSTO, Orlando fur.42 str. 81: «Fermava il pie' ciascun di questi segni / sopra due belle imagini più basse / che con la bocca aperta facean segni / che 'l canto e l'armonia lor dilettasse».

${ }^{39}$ Cf. Menge 2000, pp.114-115. Sull'inclusione della comparatio compendiaria nelle Restrictions on Coordination cf. Pinkster 1972, p.109 con i canonici esempi da Cic.De orat.1.15 e Phil.11.9.

${ }^{40}$ Giustamente il Giardina commenta: «Hoeuffti coniecturae parum mehercules credo» (cf. Giardina 1977, p.180). Il Phoebus di Houeufft trova accoglimento in Palmer ( $c f$. Smyth 1970, p.76). Poi il medesimo Hoeufft propone etiam (cf. Smyth 1970, p.76).

${ }^{41}$ La storia della trasposizione dei versi di questa elegia (di cui Giardina appare essere l'epigono) è molto antica e risale al Dousa 1592, p.111, che per ovviare al duplice riferimento alla statua di Apollo, traspone i vv. 5-8 dopo il v. 16, riferendo (non senza tautologie) il tutto al simulacro di Apollo presente nella cella. Con questa scelta ecdotica consentono Baehrens 1880, Otto 1884, p.16 e Tremenheere ( $c f$. Smyth 1970, p.75). Segue la disposizione stichica del Dousa anche l'edizione properziana di Butler 1905. Traspone i vv. 5-10 dopo il v. 16 Hertzberg ( $c f$. Smyth 1970, p.75).

${ }^{42}$ Lingua che peraltro è stata oggetto di diuturno studio da parte del Fedeli; si veda almeno Fedeli 1968 e Fedeli 1969.

${ }^{43}$ Correla la brachilogia e la comparatio compendiaria a questa figura Treves 1962, p.58. Sulla synchysis cf., inoltre, Calcante 2000, pp.22 sgg. e Torzi 2000, p.240.

${ }^{44}$ Cf. SERV.ad Aen.2.348 obscuritatem autem facit hoc loco et synchysis, id est hyperbati longa confusio.

${ }^{45} C f$. Lausberg 1969 § 334. Circa il fatto che il «linguaggio poetico» di Properzio «è caratterizzato dalla compresenza di elementi aulici ed espressivi» che «coesistono all'interno di una stessa contestualità» $c f$. Pascucci 1986, pp.199-222, part. pp.206 sgg.

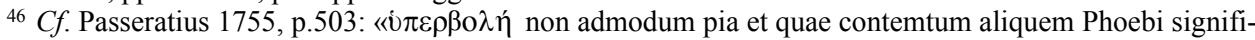
cet. Sed tunc ita solebat».

${ }^{47}$ Né escludo che Ovidio si sia ricordato di questo distico properziano, quando giocò con simile iperbole a proposito di Adone in Met.10.523 iam iuvenis, iam vir, iam se formosior ipso. 
bre Stilistica latina: «più rilevante della mera constatazione della mancanza di una parte attesa della frase, è invece la spiegazione delle circostanze che portano a modi di esprimersi ellittici. Qui occorre dapprima distinguere tra ellissi 'di risparmio' ... ed ellissi affettive, il che vale non solo per la soppressione del predicato..., ma anche per quella delle altre parti della frase», annoverando nella casistica specifica l'«omissione del sostantivo accanto a un aggettivo», fenomeno che «resta in tutta la latinità ${ }^{48}$. Insomma: se Terenzio in Ad.352 può scrivere is nostro Simulo fuit summus, sottintendendo amicus (in un contesto dove amicus peraltro non compare), se Orazio (Sat.2.7.90-91) può affermare repulsum / perfundit gelida, sottintendendo aqua, oppure limis (Sat.2.5.53) per limis oculis, perché Properzio non può aver usato marmoreus per Phoebus marmoreus vicino al nome proprio stesso che egli sottintende? Ed in proposito Hofmann-Szantyr è giustamente categorico: «Nel caso di integrazione in base al contesto... non si può parlare di vera e propria ellissi... Il fenomeno non deve stupire oltre, soprattutto là dove l'integrazione è inequivocabilmente suggerita dalla corresponsione tra le parti della frase interessate... parecchi prosatori e poeti... vanno alquanto oltre in proposito, al punto che spesso si è fatto ricorso ma per lo più senza motivo - a correzioni testuali» ${ }^{49}$.

Appartiene anche allo stile di Properzio quella «economicità di espressione» ${ }^{50}$, traducentesi in brevità, incisività ${ }^{51}$ e costrutti brachilogici, come le ellissi colloquia$\mathrm{li}^{52}$, che non si stenta di riconoscere pacificamente nello stile di Orazio.

La preziosità stilistica isola nel distico l'agg. marmoreus ed induce a focalizzare l'attenzione su di esso, che peraltro (per quanto si faccia passare quest'eco - da parte di Heyworth ${ }^{53}$ - quasi come una scoperta moderna) almeno a far data dal commento di Kuinoel (1822, p.196) costituisce parola allusiva alla descrizione del-

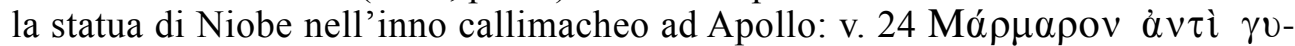

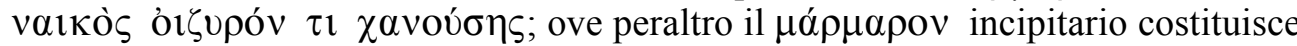
hapax (in quanto «neutrum nusquam alibi reperitur» ${ }^{54}$ ), che Valckenaer suggeriva

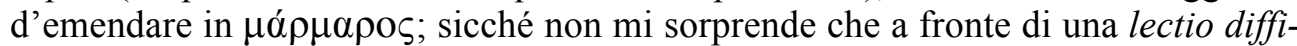
cillima del modello greco il Callimaco Romano ${ }^{55}$ abbia inteso proporre un costrutto parimenti ricercato.

I dubbi sulla bontà del testo tràdito nel v. 5 furono instillati nella filologia moderna - che dopo la gran messe di discussioni perse di vista la scaturigine della quaestio al punto da obliterarla totalmente non solo negli apparati ma anche nei commenti -

${ }^{48} C f$. Hofmann-Szantyr 2002, pp.230-231.

${ }^{49}$ Ibid. p.233. Adduco e.g. il caso interessante di Anth. Vossiana 52 a (ed. Zurli 2001), v. 5 sg. Nec licuit non nosse nefas: dum membra cruenti / nudat, dove l'agg. cruenti è riferito al sottinteso fratris, espresso nel v. 4 infelix fratris vulnere laetus erat. Lo Scaligero, non avendo compreso il riferimento suggerito invece dal contesto, inopportunamente correggeva in cruenta.

${ }^{50}$ Cf. Muecke 1997, pp.777-778 e 784, s.u. «Lingua e stile».

${ }^{51}$ Cf. Schmidt 1990, pp.57-98, part. pp.90-98.

${ }^{52}$ Cf. Ruckdeschel 1911, pp.153-158.

${ }^{53}$ Cf. Heyworth 1994, p.57.

${ }^{54} \mathrm{Cf}$. Cahen 1953, p.225.

${ }^{55}$ Cf. Giangrande 1981, pp.147-167. 
dalla prima edizione del Lachmann (Lipsiae 1816), dove il celebre filologo simpatizzò per una congettura del Markland ${ }^{56}$. E se il medesimo Lachmann già nel 1829 come s'è detto - s'era ricreduto in favore della redazione ex fide codicum, altri hanno continuato sino ad oggi a coltivare l'infelice osservazione che aveva fatto propendere per la presto rigettata hariolatio, facendone derivare i più vari interventi testuali. Nel 1816 il Lachmann (p.211) stampava in 2.31.5-6 (=3.27.5-6 della sua 'redistribuzione'):

Hic quidam Phoebo visus mihi pulchrior ipso

Marmoreus tacita carmen hiare lyra.

e commentava in relazione ad Hic equidem Phoebo che «Equidem hic plane vel profecto significare volunt, contra usum Virgilii et Ovidii et caeterorum illius saeculi», perciò giudicava da accogliersi la congettura del Markland per quanto il «Barthius quidem dubitat, an bene dicatur quidam marmoreus»; provava quindi 1'appropriatezza dell'espressione quidam marmoreus mediante rinvio al sintagma me marmoream nel v. 4 dell'epitafio n. 62 (meretricis) del IV tomo dell'Anthologia Latina del Burman $(1773, \mathrm{p} .45)^{57}$. Sull'inadeguatezza di equidem si è costruito un tale castello di elucubrazioni ${ }^{58}$ che rischia di sembrare debole ogni sua difesa; sicché non basterà osservare con Heyworth $(2009$, p.247) che «we also need to allow due weight to equidem (see $O L D 1 \mathrm{f}$ for the usage with mihi videtur): as the particle shows that aesthetic judgement is here emphasized... The Phoebus here seemed to me at any rate more beatiful than himself», ma occorrerà anche citare Hofmann-Szantyr, Postgate, Maltby ${ }^{59}$ ed elencare gli usi poetici ${ }^{60}$ di equidem con il dimostrativo ${ }^{61}$ o il determinativo ${ }^{62}$, le sue occorrenze con persone diverse dalla prima sing. ${ }^{63}$, ma so-

${ }^{56}$ Questa congettura aveva riscosso già l'approvazione del Dorvillius, in quanto si attagliava bene all'idea che la statua apollinea dei versi in esame riproducesse le fattezze di Augusto (cf. Burman 1780, p.432). Più tardi essa sarà riproposta dal Paley ( $c f$. Smyth 1970, pp.75 sg.).

${ }^{57}$ Forma atque illecebris Veneri vixi aemula Livor / Saxo delicias invidus occuluit. / Artificis pietate iterum rediviva placebam, / ni me vidisset Cypria marmoream. Lachmann avrebbe potuto citare più opportunamente Verg. Ecl.7.35 Nunc te marmoreum pro tempore fecimus; at tu, imitato da AusON. Epigr.5.1 Nunc te marmoreum pro sumptu fecimus, at cum / Augustus frater remeaverit, aureus esto, e Auson. Epigr.78.1-2 ... Ille / quamvis marmoreus.

58 Propone, ad es., di evitare equidem leggendo Hicce quidem Tremenheere (cf. Smyth 1970, p.75).

${ }^{59}$ Cf. Fedeli 2005, p.877.

${ }^{60} \mathrm{Cf}$. Quicherat 1967 s.u. «equidem».

${ }^{61}$ Cf. Plaut.Poen. 278 Hanc equidem Venerem venerabor...; Ter.Haut.709 Huic equidem consilio palmam do...; Verg.Georg.4.147 Verum haec ipse equidem; SEN.Tro.868; Phaedr. 525; Oct.540 hoc equidem; VAL.FL.Arg.3.270 hac equidem; Ermold.Pipp.1.149 Saxona hic equidem veniens de gente sagaci; Herig.Ursm.1.422 Haut secus hic equidem flatu repletus eodem; AdEMAR.Carm.5.4.1 Hic equidem genitus tumido...; Vita Gisl.415 Hic equidem cernens hic sat bonitatis amorem; 503 Hic equidem sacrum condens sub pectore verbum (con chiara eco virgiliana); YSENGRIMUS.5.964 Hic equidem.

${ }^{62}$ Cf. TER.Eun.322 Id equidem adveniens mecum stomachabar modo; Haut.632 ... Id equidem ... e VERG.Georg 4.147 cit.

${ }^{63}$ Cf. Plaut.Epid.603 Adulescentem equidem dicebant; Men.369 Quicum haec mulier loquitur? Equidem tecum; Poen.1239 sg. ...celavistis.../ atque equidem ingenuas...; Stich.554 Dum equidem hercle quod edint addas; CAECIL.Com 37 Nam nobis equidem deus repertus est Iovis; STAт.Theb.10, 198 sg.... Equidem haec... / redimus. 
prattutto $^{64}$ il suo uso enfatico, in tema di abilità nella lavorazione del marmo, nell'ambito della rassegna sulla storia futura di Roma in VERG.Aen.6.848 credo equidem vivos ducent de marmore vultus (luogo che sembra potersi correlare a Properzio e, mediante allusione, alle opere plastiche del complesso augusteo sul Palatino), ed il suo uso coevo con dimostrativo incipitario in VERG.Aen.1.238 Hoc equidem occasum Troiae tristisque ruinas (formulato e contrario in Properzio ad indicare la rinascita di Troia sul Palatino ad opera di un discendente d'Enea) e in Aen. 6.716 Has equidem memorare tibi atque ostendere coram.

La congettura di Markland aveva il pregio (agli occhi di Lachmann I, s'intende) di eliminare lo sgradito equidem e di fornire il tanto ricercato soggetto da concordare con l'agg. marmoreus. I primi ad essere stati fuorviati dalla necessità di individuare il soggetto (sottinteso) sono stati gli estensori di alcuni codices recentiores e degli Itali, i quali recano hic equidem ph(o)ebus visus... marmoreus con un evidente e vulgaris errore da omoteleuto, del tutto contrario al divieto, vigente nella poesia 'classica', di produrre l'inelegante sigmatismo, che qui sarebbe per di più accentuato dall'arsi su tutte e tre le sillabe -us (Phoebús visús... marmoreús). L'unanimemente riconosciuto campione di eleganza formale della medesima età e del medesimo genere letterario, cioè Tibullo, insegna con la sua tersa versificazione che è da rifuggire l'omoteleuto di due parole contigue e massimamente il polisigmatismo ${ }^{65}$, giudicato dalla manualistica antica cacofonico e sgraziato $^{66}$. Al che non hanno prestato forse la debita attenzione né $\mathrm{Enk}^{67}$, né il Giardina (nella sua edizione paraviana) ${ }^{68}$, né Goold ${ }^{69}$, che accolgono la lezione Phoebus visus di quei codici, né (ma con maggior cautela) il Fedeli ${ }^{70}$, che in apparato commenta con un fortasse recte il medesimo patente error vulgaris.

Stabilito il testo nei vv. 5-6 secondo la testimonianza dei codici, proviamo ora a rapportarlo alle risultanze antiquiarie ed archeologiche emerse dagli scavi sul Palatino.

Se si ammette che l'elegia presenta le opere d'arte ${ }^{71}$ secondo la progressione del percorso del poeta ${ }^{72}$, nell'area Apollinis dovevano esistere due statue di Apollo citaredo $^{73}$ : una, descritta nei vv. 5-6, esterna al tempio, nella porticus delle Danaidi, l'altra nella cella in mezzo a Latona e Diana (sì che alla triade capitolina si opponesse una triade palatina ${ }^{74}$ ), raffigurante ancora un Apollo citaredo, ma giustamente appel-

\footnotetext{
${ }^{64}$ Non mi consta che nessun commento lo abbia notato.

${ }^{65}$ Cf. Della Corte 1980, pp.310 sg. e part. Cronin 1970, pp.174-180.

${ }^{66} C$ f. Dion.Halic.De compos verb. 14.80 , p.54 Usener-Radermacher.

${ }^{67}$ Cf. Enk 1962.

${ }^{68}$ Cf. Giardina 1977.

${ }^{69}$ Cf. Goold 1990.

${ }^{70}$ Cf. Fedeli 1984.

71 Sulla poesia properziana ecfrastica di opere d'arte $c f$. Williams 2006, pp.291-314. È tornato recentemente su PROP.2.31, per i suoi riferimenti all'architettura di Roma, von Albrecht 2010.

72 Sul percorso di accesso al tempio ricostruito dalle emergenze archeologiche $c f$. Carettoni 1978, p.73. Giustamente parla di «a very well-ordered tour of the temple-complex» Hubbard 1984, p.282 e n.7.

73 Cf. Prop.4.6.69 citharam iam poscit Apollo e TiBull.2.5.2 sgg.

74 Più precisamente, Giove, Giunone e Minerva erano venerati sul Campidoglio, Cerere, Libero e Libera, sull'Aventino e Apollo, Diana e Latona sul Palatino.
} 
lato da Properzio Pythius (non più Phoebus), perché sulla base di questa statua furono collocati da Ottaviano Augusto gli oracoli sibillini.

Sappiamo da Plinio che una di queste statue apollinee, un Apollo Palatino marmoreo $^{75}$, fu opera di Scopas: Scopae laus cum his certat. is fecit Venerem et Pothon, qui Samothrace sanctissimis caerimoniis coluntur, item Apollinem Palatinum, Vestam sedentem laudatam in Servilianis hortis duosque campteras circa eam, quorum pares in Asini monimentis sunt, ubi et canophoros eiusdem (Nat.hist.36.4.25). Gli esegeti settecenteschi di Properzio ${ }^{76}$ identificavano con l'opera di Scopas 1'Apollo del portico, mentre l'esegesi moderna propende - giustamente - ad identificare con essa l'Apollo della cella del tempio, mentre prospetta che la statua dell'Apollo citaredo eretta davanti al tempio possa aver ricalcato nelle sue fattezze la statua di culto del santuario di Daphnè ad Antiochia eseguita da Briaxis ${ }^{77}$.

Ps.Acrone.ad Hor.Epist.1.3.17 ci informa che Augusto aveva fatto collocare nella biblioteca palatina, attigua alla porticus, una statua che lo raffigurava nelle fattezze di Apollo: Caesar sibi in bibliotheca statuam posuerat ad habitum et staturam ${ }^{78}$

75 Il 1. XXXVI della Naturalis Historia è dedicato alla scultura marmorea. $C f$. anche RE s.u. «Palatinus», col. 3 .

76 Cf. Passeratius 1755, p.503.

77 Cf. Gagé 1955, p.536 n. 4; Nock 1962, pp.307-310; Linfert 1983, pp.165-173 e Zanker 1989, pp.31 sgg. Dalle testimonianze letterarie e numismatiche che ce la descrivono apprendiamo che questa statua raffigurava il dio stante con la patera per le libagioni in una mano e la lira nell'altra (cf. LIBAN. Or. 61 ¿่ $\omega \dot{\kappa} \kappa \varepsilon$

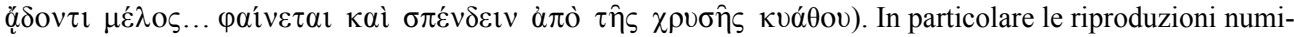
smatiche rappresentano la statua intenta a libare in prossimità di un altare. Se questa fosse la statua descritta da Properzio nei vv. 5-6, dovremmo dire che il poeta ne avrebbe omessa l'azione della libagione sostituendola con il tacita carmen hiare lyra. Ed inoltre la vicinanza della statua all'altare comporterebbe che attorno all'ara ci fossero sia Apollo sia le quattro statue di buoi, opera di Mirone (per la lezione dei vv. 7-8 cf. White 2009, pp.34 sg.): un affollamento, forse, eccessivo, ma ammesso senza alcun dubbio da Enk 1962, pp.205 sg., da Gazich 1995, p.156 e da Gros 1993, p.55. Ed allora un Apollo citaredo vicino a quattro buoi verrebbe a configurarsi soprattutto come Apollo Nomios (per questo attributo del dio cf. Daremberg-Saglio 1877 s.u. «Apollon», pp.311,313 sg.,321), la cui presenza sul Palatino si ricollegherebbe all'origine pastorale di Roma, che la Fantham (1997, p.126) mette in rilievo nell'elegia properziana 3.9, ma evocherebbe anche il luogo del Palatino dove nacque Augusto, detto ad capita Bubula (cf. Degrassi 1966-1967, p.83). Perciò quanti sostengono che 1'Apollo dei vv. 5-6 non sia lo stesso delle biblioteche, ma sia un altro Apollo di fronte al tempio e nei pressi dell'altare e dei buoi, dovrebbero anche trarne le necessarie conseguenze ideologiche, visto che esso verrebbe ad ubicarsi in corrispondenza della c.d. Roma quadrata, sito della fondazione dell'Urbe (cf. SoLIN.1.18 Roma quadrata incipit a silva quae est in area Apollinis et ad supercilium scalarum Caci habet terminum, ubi tugurium fuit Faustoli; FEsT.p.258 M. Quadrata Roma in Palatio ante templum Apollinis dicitur, ubi reposita sunt quae solent boni ominis gratia in urbe condenda adhiberi; OvID.Trist.3.1.31 inde petens dextram 'porta est' ait 'ista Palati, hic Stator, hoc primum condita Roma loco est; cf. anche Degrassi 19661967, p.95 e Thon-Ballanti 1828, pp.11 e 70) e della 'rifondazione' augustea, sotto la protezione dell'Apollo Aziaco ( $c f$. Bern 1982, pp.82-104 e Lugli 1953, pp.26-55) e al contempo Nomios. Tuttavia, i locativi hic del v. 5 e aram circum del v. 7 sembrano riferiti, nella progressione del percorso corrispondente alla progressione dei distici, a due spazi contigui sì, ma diversi.

${ }^{78}$ In merito a questa lezione il Santenius suggeriva di corregere in statu sulla base di OvID.Met.11.169 artificis status ipse fuit detto di Apollo. Ma la lezione statura trova conforto nel reperimento di alcuni frammenti di una statua colossale di Apollo sul Palatino, $c f$. Strazzulla 1990, p.133; Martin 1987, p.262 n.118, che considera i frammenti della statua colossale appartenenti alla statua di culto, e Jones Roccos 1989, p.579. Carettoni 19661967, p.73 sg., in un primo momento inferisce dalla mutilazione e dall'accanimento patito dalla statua che possa trattarsi della statua di culto, poi la connette alla statua descritta da Properzio nei vv. 5-6. 
Apollinis. Anche Servio conserva esplicita memoria di questo Apollo/Augusto: ad Verg.Buc.4.10 Tuus iam regnat Apollo. Ultimum saeculum ostendit, quod Sybilla Solis esse memoravit. Et tangit Augustum cui simulacrum factum est cum Apollinis cunctis insignibus. I medesimi esegeti settecenteschi (part. il Broukhusius) precisavano, tuttavia, che questa statua sarebbe stata diversa da quella menzionata nei vv. 56 dell'elegia. Altri esegeti, invece, come il Kuinoel (1822, p.196) sulla scia del Santenius e del Burmannus, ritennero che nei vv. 5-6 si facesse riferimento a questa statua di Apollo con il volto di Augusto ${ }^{79}$. Ed il Santenius ${ }^{80}$ precisava che appunto alla statua di Augusto/Apollo si dovessero riferire i versi virgiliani di Aen. $8.720 \mathrm{sgg}$. Ipse sedens niveo candentis limine Phoebi / dona recognoscit populorum, optatque superbis / postibus ${ }^{81}$.

La testimonianza della tabula Hebana ${ }^{82}$ ribadisce l'esistenza, in Palatio in porticu quae est ad Apollinis in eo templo in quo senatus haberi solet, di un simulacrum Apollinis, coperto da un fastigium. Poiché si sa che il senato era solito riunirsi nella zona della biblioteca, a sua volta parte del portico, il termine templum della tabula

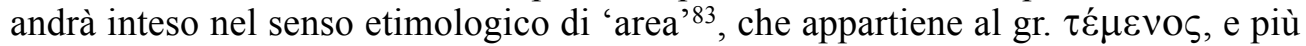

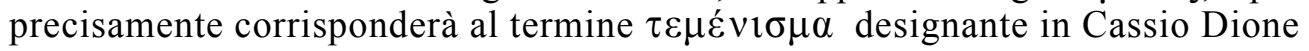
(53.1.3 cit. infra) la porticus.

Presumibilmente la statua in parola va distinta dalla imago Augusti di cui fa menzione Tacito in occasione di una seduta del senato tenutasi nella biblioteca palatina (Ann. 2.37 cum in Palatio senatus haberetur, modo Hortensii inter oratores sitam imaginem modo Augusti intuens, ad hunc modum coepit) ${ }^{84}$, e probabilmente essa va differenziata anche dalla statua colossale di Apollo fatta portare a Roma da M. Lucullo nel 73 a.C. dalla Tracia e ricordata sul Palatino da Appiano (Illyr. 80

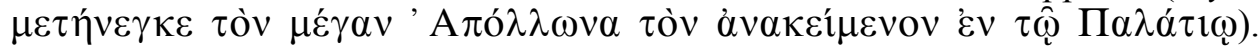

L'identificazione della statua di Apollo in porticu è strettamente connessa con l'esatta ubicazione della porticus stessa ${ }^{85}$, che sulla base della testimonianza di Velleio Patercolo ${ }^{86}$ sembrerebbe circondare il tempio di Apollo: 2.81 .3 templumque Apollinis et circa porticus facturum promisiti, quod ab eo singulari extructum munificentia est. Ma nulla vieta d'intendere l'avverbio circa nel senso generico di 'nelle vicinanze, là attorno'.

\footnotetext{
79 Sul complesso rapporto di Augusto ed Apollo e le sue implicazioni ideologiche ed iconografiche $c f$. Zanker 1989, p.54 sgg. In particolare sull'allestimento architettonico del Palatino $c f$. Zanker 1983, pp.21-40 e Winkler 2005.

${ }^{80}$ Cf. Burman 1780, p.432.

${ }^{81} \mathrm{Si}$ è pensato che Virgilio abbia scritto questi versi (desunti dalla descrizione dello scudo di Enea) posteriormente al 29 e che vi si adombrerebbero le intenzioni di Augusto in merito alla dedica di templi ed in particolare in merito all'assetto del tempio di Apollo sul Palatino. Vero è che davanti al tempio - stando all'ecphrasis virgiliana - è posto Augusto stesso, $c f$. Warde Fowler 1917, p.118 sgg.

${ }^{8}$ Cf. Last 1953, pp.27-29.

83 Cf. GELL.Noct.14.7.

${ }^{84}$ Cf. Gagé 1955, p.530 n.3.

${ }^{85} \mathrm{Su}$ questa $c f$. ultimamente Quenemoen 2006, pp.229-250, secondo la quale il portico poggiava sulla terrazza del tempio. $C f$. anche Balensiefen 1995, pp.189-209.

${ }^{86} \mathrm{Su}$ questa testimonianza $c f$. Carettoni 1966-1967, p.56.
} 
Si apprende, inoltre, da Svetonio che i portici erano tutt'uno con le biblioteche greca e latina, ivi allocate ${ }^{87}$ : Aug. 29 Publica opera plurima extruxit, e quibus vel praecipua:... templum Apollinis in ea parte Palatinae domus excitavit, quam fulmine ictam desiderari a deo haruspices pronuntiarant; addidit porticus cum bibliotheca Latina Graecaque, quo loco iam senior saepe etiam senatum habuit decuriasque iudicum recognovit.

Il che parrebbe emergere anche dalla testimonianza di Ovidio (Trist. $3.1 .59 \mathrm{sgg}$. Inde tenore pari gradibus sublimia celsis / ducor ad intonsi candida templa Dei: / signa peregrinis ubi stant alterna columnis / Belides et stricto barbarus ense pater: / quaeque viri docto veteres cepere novique / pectore lecturis inspicienda patent) $\mathrm{e}$

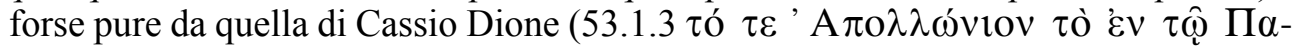

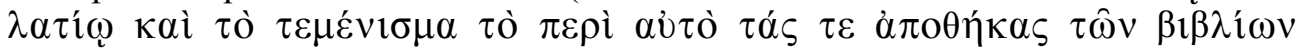

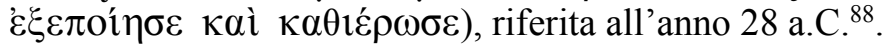

Se l'Apollo con le sembianze di Augusto è diverso da quello descritto da Properzio, si deve ammettere, allora, che nell'area Apollinis ci fossero tre statue di Apol$10^{89}$ : la statua di culto nella cella (con la lira), la statua davanti al tempio (con la lira), la statua della biblioteca (per la quale la lira del Musagete sarebbe stata idoneo attributo $^{90}$ ); il che, francamente, indurrebbe a supporre una qualche sovrapposizione.

Unico elemento ostativo all'identificazione dell'Apollo menzionato da Properzio con l'Apollo/Augusto della biblioteca in porticu sarebbe costituito da una testimonianza di Plinio ${ }^{91}$, secondo il quale la statua di Apollo sita nella biblioteca sarebbe stata di bronzo e non di marmo: Nat. hist. 34.7.34 Videmus certe Tuscanicum Apollinem in bibliotheca templi Augusti L pedum a pollice, dubium aere mirabiliorem an pulchritudine. Tuttavia va ricordato che il complesso palatino di tempio, portico e biblioteche subì un incendio nel 64 d.C. ${ }^{92}$, dopo il quale si ebbe la ricostruzione domizianea, maggiormente incentrata sulla zona delle biblioteche, che patì i danni più ingenti ${ }^{93}$. Questo dato assieme al pres. videmus della testimonianza pliniana induce ad inferire - qualora s'intenda che la biblioteca del tempio di Augusto sia quella della porticus delle Danaidi - che semmai l'Apollo bronzeo visibile al tempo di Plinio fu collocato nella zona della biblioteca dopo l'incendio stesso; ma è senz'altro più probabile che la bibliotheca templi Augusti ove fu sistemato questo Apol lo tuscanico possa essere verisimilmente quella del tempio del divus Augustus, co -

\footnotetext{
${ }^{87}$ Per quanto i dati di scavo non abbiano potuto accertare definitivamente l'ubicazione del portico delle Danaidi (cf. Tomei 2005-2006, pp.379 e 383; Quenemoen 2006 e Coarelli 2001, p.169 sg.), è indubbio che esso stesse in stretta relazione con il tempio e con i due edifici delle biblioteche, $c f$. Strazzulla 1990, p.101 sgg.; Klodt 1998, p.3. Sulla biblioteca palatina $c f$. anche Hubbard 1984.

${ }^{88}$ Data della dedica del tempio, cui si riferisce anche Hor.Carm.1.31.

${ }^{89}$ Cf. Gagé 1955, p.536 sg. e Bowditch 2009, p.411.

${ }^{90}$ Cf. Gagé 1955, p.529.

91 Testimonianza ignorata da Heyworth 1994, p.58 n.22 e sopravvalutata, un tempo, da Thon-Ballanti 1828, p. 71 .

${ }^{92}$ La porticus sarebbe stata completamente distrutta dall'incendio, $c f$. Tomei 2005-2006, p.383.

93 Cf. Gagé 1955, pp.531 e 569. Sarebbe legittimo chiedersi se non si riferisca a questa statua di Apollo/Augusto la testimonianza (su evento precedente il 64) di SVET. Nero 12.8 coronam ... citharae autem a iudicibus ad se delatam adoravit ferrique ad Augusti statuam iussit.
} 
struito sul fianco nord-occidentale del Palatino ed inaugurato nel 37 d.C. da Caligo19 ${ }^{94}$; perciò questa testimonianza pliniana con ogni probabilità non si riferisce alla statua di Apollo/Augusto di nostro interesse, che pertanto doveva essere marmorea.

Qualora, dunque, i vv. 5-6 dell'elegia properziana descrivano la statua di Apollo/Augusto ${ }^{95}$, ipotesi cui non ostano le risultanze antiquarie, si comprende ancor meglio la ragione dell'ellissi del soggetto che accompagna l'agg. marmoreus, la quale, allora, si verrebbe a configurare come una studiata, preziosa ed ammiccante reticenza del nome di un 'ibrido' artistico (ed ideologico).

In ogni caso: apprezzavano con maggior chiarezza la 2.31 i giovani lettori dei sintetici e perspicui Propertii casta carmina ad scholasticorum usum selecta ${ }^{96}$ d'un impolverato libello poetico occorsomi di sbirciare, che non i cultori contemporanei del poeta, edotti dai moderni mega-commentari properziani; perché ad un testo privo di cruces essi accedevano col supporto delle seguenti lucide note ed essenziali: «Serius se venisse dicit detentus inspectione porticus Apollinis, quae ab Augusto Caesare absoluta est. Haec porticus templo Apollinis Palatini cum Bibliotheca Graeca et Latina adiuncta erat... In illa porticu Phoebus erat marmoreus ipso Phoebo pulchrior et hiscendo carmina canere videbatur» (p.176).

Omnia nimia nocent.

\section{RIFERIMENTI BIBLIOGRAFICI}

Aken, O.A. (1884), De figurae à $\pi \dot{o}$ Kolvô̂ usu apud Catullum, Tibullum, Propertium, I, Progr., Schwerin.

Albrecht V., M. (2010), «Properz und die Architektur des augusteischen Rom», in corso di stampa in Properzio fra tradizione e innovazione, «Atti del Convegno internazionale Accademia Properziana, Assisi-Spello 21-23 maggio 2010».

Amar, J.A. (1821), Sexti Aurelii Propertii Carmina ex Christ. Theoph. Kuinoel recensione, Parisiis.

Baehrens, Ae. (1880), Sexti Propertii Elegiarum libri IV, Leipzig, Teubner.

BALENSIEFEN, L. (1995), «Überlegungen zu Aufbau und Lage der Danaidenhalle auf dem Palatin», $\operatorname{MDAI}(R)$ 102, 189-209.

Barber, E.A. (1953), Sexti Properti Carmina, Oxonii, e Typogr. Clarendon.

Barber, E.A. $\left(1960^{2}\right)$, Sexti Properti Carmina, Oxonii, e Typogr. Clarendon.

BARCHIESI, A. (2005), «Learned Eyes: Poets, Viewers, Image Makers», in GALINSKY, K. (ed.), The Cambridge Companion to the Age of Augustus, Cambridge, Cambridge University Press, pp.281-305.

${ }^{94}$ Cf. Gagé 1955, p.575 sg.

${ }^{95}$ Sembra propendere per l'identificazione Barchiesi 2005, pp.281-305.

${ }^{96}$ Cf. C. Valerii Catulli Albii Tibulli et Sexti Aurelii Propertii casta carmina ad scholasticorum usum selecta, notisque extemporalibus illustrata nova editio auctior et emendatior cui adnotationes et dilucidationes accedunt Cl. Vuplii commentariis, Bassani 1824. 
BERN, H.J. (1982), «Apollo Palatinus und Apollo Actius auf augusteischen Münzen», MH 39, 82-104.

Bonazzi, G. (1939), Le elegie di Sesto Properzio secondo la lezione genuina conservata dal manoscritto vaticano "Palatino Latino 910» con traduzione metrica, Roma, Signorelli.

BonazzI, G. (1951), Propertius resartus elegiarum libri a diuturna interpolatione redempti, Romae, Bretschneider.

Bowditch, L. (2009), «Palatine Apollo and the imperial gaze: Propertius 2.31 and 2.32», AJPh 180, 401-438.

Broukhusius, J. (1753), Tibulli et Propertii opera ex editione J. Broukhusius fideliter expressa, Glasguae.

Burman, P. (1773), Anthologia veterum Latinorum epigrammatum et poematum, t. II, Amstelaedami, ex officina Schouteniana.

Burman, P. (1780), Sex. Aurelii Propertii elegiarum libri IV cum commentario perpetuo Petri Burmanni Secundi et multis doctorum notis ineditis, Trajecti ad Rhenum, apud Barth. Wild.

Butler, H.E (1905), Sexti Properti opera omnia, London, Archibald Constable.

Butler, H.E.-BARBer, E.A. (1969), The Elegies of Propertius, ed. with an introd. and comm., Hildesheim-New York, rist.

Cahen, E. (1953), Callimaque, Paris, Les Belles Lettres.

CAlcante, C.M. (2000), Genera dicendi e retorica del sublime, Pisa-Roma, Istituti editoriali e poligrafici internazionali.

CAmps, W.A. (1967), Elegies. Book II, Cambridge, Cambridge University Press.

Carettoni, G. (1978), «Roma - Le costruzioni di Augusto e il tempio di Apollo sul Palatino», QuadAEI 1, 72-74.

Carettoni, G. (1966-1967), «I problemi della zona augustea del Palatino alla luce dei recenti scavi», RendPontAcc 39, 55-75.

Clyde, J. (1856), Greek Syntax with a Rationale of the Constructions, Edimburgh-London, Edimburgh: Sutherland and Knox, London: Simpkin, Marshall and Co.

CoArelli, F. (2001), Roma, nuova ediz., Roma-Bari, Laterza.

Cronin, P.A. (1970), «Sigmatism in Tibullus and Propertius», CQ 20, 174-180.

Daremberg, Ch.-SAglio, E. (1877), s.u. «Apollon», Dictionnaire des antiquités grecques et romaines, Paris, Hachette.

Davies, G.A.T. (1907), p.Ovidi Nasonis Metamorphoseon liber XI, Oxford, Clarendon Press.

Degrassi, N. (1966-1967), «La dimora di Augusto sul Palatino e la base di Sorrento», RendPontAcc 39, 77-116.

Della Corte, F. (1980), «Versificazione e metrica verbale», in Appendice a Tibullo. Le elegie, Milano, Mondadori.

Dousa, I. (1592), Iani Dousae filii In Catullum, Tibullum, Propertium coniectanea et notae, Lugduni Batavorum, ex Officina Plantiniana.

ENK, P.J. (1962), Sex. Propertii Elegiarum liber secundus cum prolegomenis, conspectu librorum et comm. Ad IV libros Prop.pertinentium ..., Lugduni Batavorum, in aedibus A.W. Sijthoff.

FAntham, E. (1997), «Images of the city: Propertius' new-old Rome», in HABINEK, Th.Schiesaro, A. (ed.), The Roman Cultural Revolution, Cambridge, Cambridge University Press, pp.122-135.

FARRAR, F.W. (1867), A brief Greek Syntax and hints on Greek Accidence, with some reference to comparative philology and with illustrations from various modern languages, London, Longmans, Green. 
Fedeli, P. (1968), «Osservazioni sullo stile di Properzio», in Omaggio a Eduard Fraenkel per i suoi ottant'anni. Contributi di allievi dei suoi seminari di Pisa, Bari e Roma, Roma, pp.88-106.

Fedeli, P. (1969), «Osservazioni sullo stile di Properzio», SIFC 41, 81-94.

Fedeli, P. (1984), Sexti Properti Elegiarum libri IV, Stutgardiae, Teubner.

Fedeli, P. (2005), Properzio Elegie libro II, introd. testo e comm., Cambridge, Francis Cairns.

Fusi, A. (2006), M. Valerii Martialis Epigrammaton liber tertius, Hildesheim-Zürich-New York, Olms.

GAGÉ, J. (1955), «Apollon Romain», BEFAR 182, 523-581.

Galli, D. (2007), Valerii Flacci Argonautica I. Commento, Berlin, de Gruyter.

Gazich, R. (1995), 'Exemplum'ed esemplarità in Properzio, Milano, Vita e Pensiero, Pubblicazioni dell'Università Cattolica.

Giangrande, G. (1981), «Propertius Callimachus Romanus», in Colloquium Propertianum II, Assisi, La Porziuncola, pp.147-167.

Giardina, G. (1977), Sex. Properti elegiarum liber II, edidit brevi commentario instruxit Ioannes Carolus Giardina, Aug. Taurinorum-Mediolani, Paravia.

Giardina, G. (2005), Elegie. Properzio, Roma, Ed. dell'Ateneo.

Gros, P. (1993), «Apollo Palatinus», in Lexicon Topographicum Urbis Romae, vol. I, Roma, Quasar.

Goold, G.P. (1990-1999²), Propertius. Elegies, Cambridge/Mass., Harvard University Press.

Gudeman, A. (1914), P. Cornelii Taciti Dialogus de oratoribus, Leipzig, rist. Amsterdam 1967.

Hanslik, R. (1979), Sex. Propertii elegiarum libri IV, Leipzig, Teubner.

Haupt, M.H.-Ehwald, R.H. (19159), P. Ovidius Naso, Metamorphosen, I: Buch I-VII, Berlin.

Heyworth, S.J. (1994), «Some allusions to Callimachus in Latin poetry», MD 33, 56-59.

Heyworth, S.J. (2007), Sexti Properti Elegos, Oxford, e Typogr. Clarendon.

Heyworth, S.J. (2009), Cynthia. A Companion to the text of Propertius, Oxford, Oxford University Press.

Hofmann, J.B.-Szantyr, A. (2002), Stilistica latina, a cura di TrainA, A., trad. di Neri, C., aggiornamenti di ONIGA, R., revisione e indici di PIERI, B., Bologna, Pàtron.

HubBard, Th.K. (1984), «Art and Vision in Propertius 2.31/32», TAPhA 114, 218-297.

IACOB, F. (1827), Sex. Aurelii Propertii Carmina ad fidem optimorum codicum recensuit, integram Groningani, Neapolitani, excerptorum Puccii varietatem lectionis brevemque adnotationem adiecit F. Iacob, Lipsiae, Teubner.

Jones Roccos, L. (1989), «Apollo Palatinus: the Augustan Apollo on the Sorrento Base», AJA 93, 577-579.

Katz, V. (2004), The complete Elegies of Sextus Propertius, Princeton, Princeton University Press.

Kleywegt, A.J. (1986), «Die Dichtersprache des Valerius Flaccus», $A N R W$ 32/4, pp.24482490.

KLodT, C. (1998), «Platzanlagen der Kaiser in der Beschreibung der Dichter», Gymnasium $105,1-38$.

KortekaAs, G.A.A. (2007), Commentary on the Historia Apollonii regis Tyri, Leiden, Brill.

KROLl, W. (1924), «Die Dichtersprache», in Studien zum Verständnis der römischen Literatur, Stuttgart, Metzler, pp.247-279. 
Kroll, W. (1988), «La lingua poetica romana», in Lunelli, A. (ed.), La lingua poetica latina, Bologna, Pàtron, pp.1-66.

Kuinoel, Ch.Th. (1822), Sexti Aurelii Propertii Carmina quae extant ex recensione Ch.Th. Kuinoel, t. I, Augustae Taurinorum, ex typis viduae Pomba et filiorum.

KÜHNER, R. (1878), Grammatik der Lateinischen Sprache, Hannover, Hahn.

Lachmann, C. (1816), Sex. Aurelii Propertii carmina. Emendavit ad codicum meliorum fidem et annotavit Carolus Lachmannus, Lipsiae, apud Gerhard Fleischer Jun.

Lachmann, C. (1829), Sex. Aurelii Propertii Elegiae ex recognitione Caroli Lachmanni, Berolini, typis impensis Ge. Reimeri.

La Penna, A. (1969), Orazio. Le opere. Antologia, Firenze, La Nuova Italia.

LASt, H. (1953), «The Tabula Hebana and Propertius 2, 31», JRS 43, 27-29.

LAusBerg, H. (1969), Elementi di retorica, trad. it., Bologna, Il Mulino.

Linfert, A. (1983), «Der Apollo von Daphne des Briaxis», DamM 1, 165-173.

Luck, G. (1996), Liebeselegien: lateinisch und deutsch. Properz und Tibull; neu hrsg. und übers., München-Zürich, Artemis und Winkler.

Lugli, G. (1953), «Il tempio di Apollo Aziaco e il gruppo augusteo sul Palatino», AnnAccSanLuca 1, 26-55.

MAriotti, S. (2000), Scritti di filologia classica, Roma, Salerno Editrice.

MARTIN, H.G. (1987), Römische Tempelkultbilder, Roma, L'Erma di Bretschneider

Martini, M.M. (1931), Sexti Properti Elegiarum libri. Properzio le elegie, Milano, Società An. Notari.

Menge, H. (2000), Lehrbuch der lateinischen Syntax und Semantik, Darmstadt, Wissenschaftliche Buchgesellschaft.

Middleton, G. (1892), An Essay on Analogy in Syntax illustrated chiefly from the Classical Languages with an Appendix containing the Istances of synctactical Analogy peculiar to Herodotus, London, Longmans, Green.

Moorhouse, A.CH. (1982), The Syntax of Sophocles, Leiden, Brill.

MueCKe, F. (1997), s.u. «Lingua e stile», Enciclopedia oraziana, vol. II, Roma, Treccani.

Mueller, L. (1874), Catulli Tibulli Propertii carmina accedunt Laevii Calvi Cinnae aliorum reliquiae et Priapea, rec. et praefatus est L. Mueller, Lipsiae, Teubner.

Mueller ${ }^{1}$, L. (1874), Sex. Propertii Elegiae, rec. L. Mueller, Lipsiae, Teubner.

Mueller, L. (1885), Sex. Propertii Elegiae, rec. L. Mueller, Lipsiae, Teubner

Namia, G. (1973), Opere di Albio Tibullo e Sesto Properzio, Torino, UTET.

Nethercut, W.R. (1983), «Recent Scholarship on Propertius», ANRW II 30/3, pp.18131857.

NisARD, D. (1883), Oeuvres complètes d'Horace, de Juvenal, de Perse, de Sulpicia, de Turnus, de Catulle, de Properce, de Gallus et Maximien, de Tibulle, de Phedre, Paris, Firmin Didot.

Nock, A.D. (1962), «Sapor I and the Apollo of Briaxis», AJA 66, 307-310.

Oтто, A. (1884), Versumstellungen bei Properz, I Progr. Kath. Gymn. Gross-Glogau.

PAsCuCCI, G. (1986), «Il callimachismo stilistico di Properzio», in Bimillenario della morte di Properzio, «Atti del Convegno internazionale di studi properziani», Assisi, La Porziuncola, pp.199-222.

Passeratius, J. (1755), Sex. Aurelius Propertius Vmber et in eum Johannis Passeratii Praelectiones solemnes, sive commentarii: Jani Broukhusii notae selectae: Joannis Antonii Vulpii animadversiones perpetuae atque indices locupletissimi: omnia ex accurata eiusdem Vulpii recensione, Patavii, excudebat Josephus Cominus. 
Pinkster, H. (1972), On latin Adverbs, Amsterdam, North-Holland Publishing Company, rist. Amsterdam University Press 2005.

Quenemoen, C. (2006), «The Portico of the Danaids: a new Reconstruction», AJA 110, 229250.

QuicherAt, L. (1967), Thesaurus poeticus Linguae Latinae, rist. Hildesheim, Olms.

Ramorino, F. (1912), C. Valerii Catulli Albi Tibulli Sex. Propertii Carmina, Florentiae.

Ramorino, F. (1915), C. Valerii Catulli Albi Tibulli Sex. Propertii Carmina, Florentiae.

Riesenweber, TH. (2007), Uneigentliches Sprechen und Bildermischung in den Elegien des Properz, Berlin, de Gruyter.

Rothstein, M. (1920²), Die Elegien des Sextus Propertius, I-II, Berlin, Weidmann.

Rothstein, M. (1966), Propertius Sextus Elegien, erkl. von M. Rothstein, Dublin-Zürich 1966, rist., Weidmann.

Ruckdeschel, F. (1911), Archaismen und Vulgarismen in der Sprache des Horaz, diss. Erlangen.

Scaliger, Io. (1577), Catulli Tibulli Properti nova editio, Iosephus Scaliger recensuit, Lutetiae, apud Mamertum Patissonium.

SCHMIDT, R.A. (1990), «Schema Horatianum», WS 103, pp.57-98.

Schuster, M.-Dornseiff, F. (1958), Sex. Propertii elegiarum libri IV, ed. altera, Lipsiae, Teubner.

ScIvoletto, N. (2000), Opere di Publio Ovidio Nasone, vol. III, Metamorfosi, Torino, UTET.

Smyth, W.R. (1970), Thesaurus criticus ad Sexti Propertii textum, Lugduni Batavorum, Brill.

Strazzulla, M.J. (1990), Il principato di Apollo. Mito e propaganda nelle lastre «Campana» dal tempio di Apollo Palatino, Roma, L'Erma di Bretschneider.

Thon, C.-Ballanti, V. (1828), Il palazzo dei Cesari sul Palatino, Roma, Società tipografica.

TomeI, M.A. (2005-2006), «Danaidi in rosso antico dal Palatino», MDAI(R) 112, 379-384.

Torzi, I. (2000), Ratio et usus. Dibattiti antichi sulla dottrina delle figure, Milano, Vita e Pensiero, Pubblicazioni dell'Università Cattolica.

Treves, P. (1962), Lo studio dell'antichità classica nell'Ottocento, Milano-Napoli, Ricciardi.

VIARRE, S. (2005), «Réflexions sur les tendances actuelles de l'édition de Properce: à propos de G.P. Goold, Loeb Classical Library 1990 et $1999^{2} \gg$, in Poignault, R. (ed.) Présence de Catulle et des élégiaques latins, «Actes du colloque tenu à Tours (28-30 novembre 2002)» Clermont-Ferrand, pp.491-499.

Viarre ${ }^{1}$, S. (2005), Properce. Élégies, Paris, Les Belles Lettres.

Vulpius, J.A. (1755), Sex. Aurelius Propertius Umber et in eum Joannis Passeratii Praelectiones solemnes, sive commentarii aliquantum contracti Jani Broukhusii notae selectae Joannis Antonii Vulpii animadversiones perpetuae atque indices locupletissimi Omnia ex accurata eiusdem Vulpii recensione, Patavii, excudebat Josephus Cominus.

Warde Fowler, W. (1917), Aeneas at the Site of Rome, Oxford, B.H. Blackwell.

White, H. (2009), «Further Notes on Propertius Book II», Parnassos 2009, 31-38.

Williams, M.F. (2006), «Propertius on Art (Prop III, 9, 9-16; II, 3, 41-44; II, 6, 27-34; II, 12, 1-12; II, 31): Epigram, Aristotle, and the New Posidippus (P. Mil. Vogl. VIII, 309, Pos. X, 8-XI, 5 Bastianini = 62-70 AB)», in Deroux, C. (ed.), Studies in Latin Literature and Roman History, Bruxelles 2006, Coll. Latomus, pp.291-314.

Winkler, C. (2005), Aurea templa: der Apollo-Palatinus- und der Apollo-Sosianus- Tempel in Rom, Leipzig, Univers. Leipzig. 
ZANKer, P. (1983), «Der Apollontempel auf dem Palatin. Ausstattung und politische Sinnbezüge nach der Schlacht von Actium», in DE FINE LICHT, K. (ed.), Città e architettura nella Roma imperiale, «AnalRom», Suppl. 10, pp.21-40.

ZANKer, P. (1989), Augusto e il potere delle immagini, trad. it., Torino, Einaudi.

Zurli, L. (2001), Anthologia Vossiana, Roma, Herder Editrice e Libreria.

Zurl, L.-Mastandrea, P. (2009), Poesia latina, nuova e-filologia. Opportunità per l'editore e per l'interprete, "Atti del Convegno internazionale, Perugia, 13-15 settembre 2007», Roma, Herder Editrice e Libreria. 